\title{
Daños para la salud tras exposición laboral a nanopartículas
}

\section{Adverse health effects due to occupational exposure to nanoparticles}

\author{
Lydia Gutiérrez González 1, 2, María José Hernández Jiménez 1, 2, Leonor Molina Borchert 1, 2 \\ 1. Unidad Docente Medicina de Trabajo. Navarra. España. \\ 2. Escuela Nacional de Medicina del Trabajo. Instituto de Salud Carlos III. Madrid. España \\ Recibido: 12-04-13 \\ Aceptado: 31-05-13

\section{Correspondencia} \\ Leonor Molina Borchert \\ Instituto Navarro de Salud Laboral \\ Pol. de Landaben Calle E 31012 Pamplona \\ Tfno.: 848423700 \\ Correo electrónico: leonor.molina6@gmail.com
}

Resumen

Introducción: La exposición a nanopartículas ha aumentado en los últimos años de forma significativa debido a su utilización en muchos sectores industriales y en el ámbito doméstico. Se prevé que el empleo en la industria de la nanotecnología aumente hasta alcanzar los 10 millones de puestos de trabajo en el mundo. A pesar del número creciente de industrias y trabajadores dentro del sector, todavía no existen muchos estudios que aborden aspectos toxicológícos, la vigilancia de la salud y la higiene industrial en el sector de la nanotecnología.

Objetivo: Revisar la literatura científica reciente buscando evidencias sobre posibles efectos tóxicos y daños sobre la salud tras exposición laboral a nanopartículas.

Metodología: Se realizaron búsquedas bibliográficas en las siguientes bases de datos bibliográficas: MEDLINE (PUBMED), OSH UPDATE, IBECS, LILACS, SCIELO y CISDOC. Se revisó la literatura científica en busca de posibles efectos en la salud de la exposición a las nanopartículas.

Resultados: Se incluyeron en el estudio un total de 11 artículos científicos que cumplian los requisitos y analizaban los efectos de la toxicidad tras exposición a nanopartículas en poblaciones humanas, 4 sobre toxicidad respiratoria, 2 dermatológica, 3 de ambas exposiciones en la misma muestra y 2 estudios emulando en laboratorio exposiciones a partículas ultrafinas en ambiente laboral.

Conclusiones: Aunque no existe mucha literatura científica que estudie este tipo de relación y se hace necesario promover más estudios que profundicen sobre la materia, los trabajos existentes apuntan a que puede existir alguna relación entre la exposición a nanopartículas y problemas de toxicidad respiratoria y/o dermatológica.

Med Segur Trab (Internet) 2013; 59 (231) 276-296

Palabras claves: Nanoparticles, Nanotechnology, Occupational Health, Occupational Disease, Riesgos Laborales, Nanocomposite, Nanoclay, Nanospheres, Fullerenes, Carbon Nanotubes.

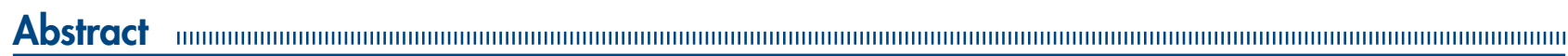

Introduction: Exposure to nanoparticles has increased in recent years significantly due to its use in many industries and in the home. It is expected that employment in the nanotechnology industry will increase to 10 million jobs in the world. Despite the growing number of nanoparticles-related industries and 
workers, there are very few published studies that address toxicity, health surveillance and industrial hygiene in the field of nanotechnology.

Objective: To review recent scientific literature for evidence on possible toxic effects and health damage after occupational exposure to nanoparticles.

Methods: We searched the literature for the following bibliographic databases: MEDLINE (PubMed), OSH UPDATE, IBECS, LILACS, SciELO and CISDOC. Scientific literature was reviewed for possible health effects of exposure to nanoparticles.

Results: We found 11 scientific articles that met the requirements and analyzed the effects of toxicity following exposure to nanoparticles in human populations, 4 on respiratory toxicity, 2 on dermatologic toxicology, 3 on both (respiratory and dermatologic on the same sample), and 2 laboratory studies simulating exposure to ultrafine particles in the workplace.

Conclusions: Despite the lack of literature on this topic and the need for more studies to go deeper into this subject, available studies suggests that there may be some relationship between exposure to nanoparticles and respiratory and/or dermatology toxicity.

Med Segur Trab (Internet) 2013; 59 (231) 276-296

Key words: Nanoparticles, Nanotechnology, Occupational Health, Occupational Disease, Riesgos Laborales (Occupational Risks,) Nanocomposite, Nanoclay, Nanospheres, Fullerenes, Carbon Nanotubes. 


\section{INTRODUCCIÓN}

La nanotecnología es la ciencia que estudia el desarrollo de materiales, dispositivos y sistemas de tamaño nanométrico, proporcionando productos con nuevas propiedades fisicoquímicas diferentes a las de las moléculas individuales o sólidos de la misma composición $^{1}$.

Se entiende por nanómetro, la unidad de longitud equivalente a una milmillonésima parte de un metro $\left(1 \mathrm{~nm}=10^{-9} \mathrm{~m}\right)$, es decir, $1 \mathrm{~mm}$ equivale a 1 millón de $\mathrm{nm}$. En general, las nanopartículas (NPs) son partículas con una o más dimensiones del orden de 100 nm o menos.

Podemos considerar que de forma natural nos encontramos rodeados de miles de millones de NPs, incluyendo el polvo de arena, las sustancias químicas generadas por el plancton oceánico, los productos de combustión (principalmente los gases de combustión de los motores diesel), procesos naturales (volcanes $\mathrm{e}$ incendios han generado nanopartículas) y otras nanoestructuras que permanecen en el ambiente a lo largo del tiempo. Sin embargo, los avances alcanzados en los últimos años con el descubrimiento de nuevos materiales y nuevos productos de aplicación industrial y doméstica, han llevado consigo un incremento importante del nivel de exposición dentro de todos los ambientes sociales, pero especialmente dentro del ámbito laboral relacionado con sectores como la electrónica, óptica, industria textil, alimentaria, de producción de pinturas, catalizadores químicos, fármacos, biomedicina, cosméticos y cremas solares, principalmente y entre otros muchos.

Se prevé que el empleo industrias de nanotecnología aumente hasta alcanzar los 10 millones de puestos de trabajo en todo el mundo en 2014 , lo que supondría un $11 \%$ de de los puestos de trabajo del sector de la industria de manufacturación. Esto significa que en la actualidad, trabajarían en Europa unos 6 millones de personas en el sector de la nanotecnología.

Uno de los problemas que nos encontramos, debido a lo novedoso de esta tecnología, es la necesidad de normalizar la terminología, por lo que diferentes instituciones europeas dedicadas a la normalización, tanto de ámbito nacional como internacional, han trabajado y creado diferentes comités de normalización ${ }^{7}$ :

— ISO TC229 "nanotechnologies"

- CEN TC 352 "nanotechnologies"

- IEC/TC 113

- OECD Grupo de Trabajo de nanomateriales fabricados.

- REACH: Reglamento (CE) 1907/2006 Relativo al registro, la Evaluación, la Autorización y la restricción de sustancias químicas y preparados. No es específico pero los nanomateriales entran bajo la definición de "sustancia" REACH. Aunque deberían ser incluidas como sustancias altamente preocupantes y (de acuerdo con el principio de precaución) evaluar adecuadamente sus efectos sobre la salud y el medio ambiente, antes de su uso y comercialización, ya que dichos efectos se desconocen.

- La Comisión Europea también ha abordado la problemática de los riesgos asociados a las nanotecnologías. (Comisión Europea, 11-12-2012). (http://ec.europa.eu/nanotechnology/faq/faqs.cfm?lg=es\&sub=results)

- La OECD (Organization for Economic Co-operation and Development) tiene una lista de los 14 nanomateriales más representativos que incluye:

1. Fullerenos (C60).

2. Nanotubos de carbono de pared simple (SWCNTs).

3. Nanotubos de carbono de pared múltiple (MWCNTs).

4. Nanopartículas de plata.

5. Nanopartículas de hierro. 

6. Carbón negro.
7. Dióxido de titanio.
8. Óxido de aluminio.
9. Óxido de cerio.
10. Óxido de cinc.
11. Dióxido de Silicio.
12. Poliestireno.
13. Dendrímeros.
14. Nano-arcillas.

La especificación técnica ISO TS 27687:2008 del Institutio Nacional de Seguridad e Higiene en el Trabajo (INSHT) de España, establece la siguiente clasificación y estructura jerárquica para la NPs (Figura 1):

Nano-objeto: material cuyas dimensiones externas o estructura interna se hallan en la nanoescala (escala nanométrica), y que puede mostrar caraterísticas nuevas comparadas con las del mismo material fuera de la nanoescala.

Nanopartícula: nano-objeto con las tres dimensiones en la escala nano.

Nanoplato: nano-objeto con una dimensión en escala nano y otras dos significativamente más largas.

Nanofibra: nano-objeto con dos dimensiones en escala nano y la otra significativamente más larga.

Nanotubo: nanofibra hueca.

Nanocable: nanofibra conductora o semiconductora de la corriente.

Nanovarilla: nanofibra sólida y recta.

Figura 1. Clasificación de las NP según la ISO TS 27687

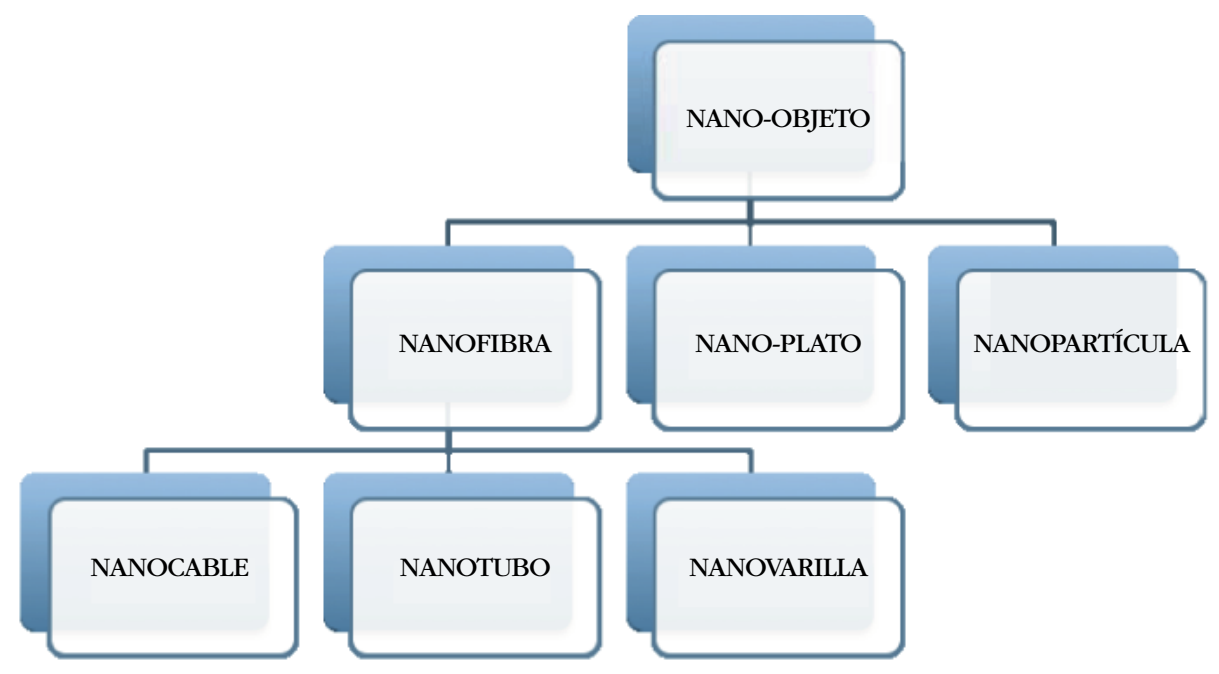

(Reproducida de Celia Tanarro Gozalo y Virginia Galvez Pérez. Centro Nacional de las nuevas tecnologías. Instituto Nacional de Seguridad e Higiene en el Trabajo (INSHT). Madrid. España. Revista Seguridad y Salud en el Trabajo).

Aunque no existe una clasificación única de NPs la mayoría de los autores hacen referencia a ellas en función de su origen dividiendo en ${ }^{5}$ :

\section{Nanopartículas de origen natural}

Algunas son de origen biológico, como por ejemplo muchos virus y bacterias. Otras son de origen mineral o medioambiental, como las que contiene el polvo de arena del desierto o las nieblas y humos derivados de la actividad volcánica o de los fuegos forestales. 


\section{Nanopartículas generadas por la actividad humana}

Las NPs consecuencia de la actividad humana pueden ser generadas de forma involuntaria o deliberada.

- Las nanopartículas producidas de forma involuntaria son las que se producen en ciertos procesos industriales; como la pirolisis a la llama del carbón negro, la producción de materiales a gran escala por procedimientos a altas temperaturas (como el humo de sílice, partículas ultrafinas de óxido de titanio y metales ultrafinos), los procesos de combustión (diesel, carbón), la obtención de pigmentos, o los procesos domésticos (barbacoas, humos de aceite). Estas nanopartículas también se llaman partículas ultrafinas (PUFs).

- Las nanopartículas generadas deliberadamente se producen mediante las llamadas nanotecnologías. En este caso la exposición se puede dar en todas las etapas de producción, desde la recepción y almacenamiento de materias primas, hasta el envasado y transporte de productos terminados (Tabla I). Los métodos para la obtención de nanopartículas son, a grandes rasgos, de dos tipos: los llamados "top-down", en los que se llega a nanomateriales sometiendo materiales convencionales a diversos procesos y los "bottom-up" en los que se construyen nanopartículas a partir de átomos o moléculas.

Tabla I. Ámbitos industriales en los que se pueden generar nanopartículas

\begin{tabular}{ll}
\hline \multicolumn{1}{c}{ Sector de actividad } & \multicolumn{1}{c}{ Ejemplos de aplicaciones actuales } \\
\hline $\begin{array}{l}\text { Automoción } \\
\text { y aeronáutica }\end{array}$ & $\begin{array}{l}\text { Materiales reforzados más ligeros; pinturas más brillantes, con efectos de color, } \\
\text { resistentes a arañazos, anticorrosiones y anti-incrustantes; aditivos para diesel que } \\
\text { permiten una mejor combustión; neumáticos más duraderos y reciclables. }\end{array}$ \\
\hline $\begin{array}{l}\text { Electrónica } \\
\text { comunicación }\end{array}$ & $\begin{array}{l}\text { Memorias de alta densidad y procesadores miniaturizados, células solares, } \\
\text { tecnología inalámbrica, pantallas planas. }\end{array}$ \\
\hline Química y materiales & $\begin{array}{l}\text { Pinturas anticorrosión e ignifugas, catalizadores, textiles con recubrimientos } \\
\text { antibacterianos y ultrarresistentes, materiales más ligeros y resistentes. }\end{array}$ \\
\hline Farmacia, biomedicina & $\begin{array}{l}\text { Medicamentos a medida liberados en órganos específicos, kits de autodiagnóstico, } \\
\text { biosensores, prótesis, implantes. }\end{array}$ \\
\hline bosmética & Cremas solares transparentes, nanoesferas con antioxidantes. \\
\hline Salud & $\begin{array}{l}\text { Dispositivos de diagnósticos y detección miniaturizados, destrucción de tumores } \\
\text { por calor, terapia génica, microcirugía y medicina reparadora, nanoimplantes y } \\
\text { prótesis, membranas para diálisis. }\end{array}$ \\
\hline Energía & $\begin{array}{l}\text { Generación de energía fotovoltaica, nuevos tipos de baterías, ventanas inteligentes, } \\
\text { materiales aislantes más eficaces. }\end{array}$ \\
\hline Medio ambiente & $\begin{array}{l}\text { Disminución de las emisiones de CO2, producción de agua ultrapura a partir de } \\
\text { agua de mar, pesticidas y fertilizantes más eficaces y menos dañinos; sensores para } \\
\text { y ecología }\end{array}$ \\
\hline la limpieza del medio ambiente.
\end{tabular}

Los nanomateriales también se pueden clasificar según su forma ${ }^{6}$ o según el número de dimensiones que en la estructura tengan carácter nanométrico :

- Tres dimensiones a escala nanométrica: nanocristales, fullerenos y puntos cuánticos.

- Dos dimensiones a escala nanométrica: nanotubos y los nanohilos.

- Una dimensión a escala nanométrica: estructuras que se utilizan en los recubrimientos de superficies o películas finas en los que sólo su grosor es de orden nanométrico. 


\section{Fullerenos}

Son estructuras cerradas formadas por átomos de carbono dispuestos en forma de pentágonos y hexágonos. Son capaces de resistir presiones extremas y de recuperar su forma original cuando cesa la presión. Se usan como lubricantes, catalizadores, semiconductores y combinados con nanotubos de carbono en nanofarmacología para "targeting" farmacológico.

\section{Puntos cuánticos}

Son un conjunto de materiales entre los 2 a $10 \mathrm{~nm}$ que pueden estar compuestos de metales o materiales semiconductores. Ofrecen propiedades electrónicas, ópticas, magnéticas y catalíticas. Tienen la capacidad de dar lugar a diferentes colores de luz, los cuales se logran modificando el tamaño de los nanocristales individuales. Esta propiedad permite que se utilicen en técnicas para el mercado fluorescente en aplicaciones biomédicas.

\section{Nanotubos de carbono}

Pueden definirse como un tubo cuya pared es una malla de agujeros hexagonales. Es posible obtener nanotubos de carbono (CNT) de varias capas, a modo de varios tubos concéntricos. Son grandes superconductores capaces de resistir el paso de corrientes elevadísimas y, por otra parte, su resistencia mecánica es sesenta veces superior a la de los mejores aceros, a su vez son ligeros y flexibles, ya que su peso específico es más de seis veces inferior a la de los mencionados aceros. También es de destacar la estabilidad de sus propiedades térmicas y químicas. Los nanotubos tienen la propiedad de emitir luz y la capacidad de convertir señales electrónicas en ópticas muy eficientemente.

\section{Grafeno}

En grafeno los átomos de carbono se unen en láminas planas de un átomo de espesor. El grafeno es un semiconductor. Este material ha llegado para revolucionar la electrónica permitiendo fabricar dispositivos electrónicos mucho más pequeños que cualquiera de los que existen actualmente.

Una última clasificación de los nanomateriales ${ }^{2}$ es la que se aprecia en la tabla II:

Tabla II. Ejemplos de Nanomateriales de $1^{\mathrm{a}}, 2^{\mathrm{a}}$ y $3^{\mathrm{a}}$ generación

\begin{tabular}{lll}
\hline \multicolumn{1}{c}{$\begin{array}{c}\text { Primera generación. } \\
\text { Nanomateriales pasivos }\end{array}$} & \multicolumn{1}{c}{$\begin{array}{c}\text { Segunda generación. } \\
\text { Nanomateriales activos }\end{array}$} & $\begin{array}{c}\text { Tercera y Cuarta generación. } \\
\text { Nanosistemas }\end{array}$ \\
\hline Carbón negro & $\begin{array}{l}\text { Funcionalizadas: CNT, nanoAg, } \\
\text { nanoAu, dendrímeros, puntos } \\
\text { cuánticos }\end{array}$ & Nanomedicinas multifuncionales \\
\hline Carbón nanotubos & $\begin{array}{l}\text { Componentes activos nanoescala } \\
\text { de circuitos integrados }\end{array}$ \\
\hline NanoTiO2 & \\
NanoSiO2 & \\
\hline NanoAg & \\
\hline
\end{tabular}

Salvando las dificultades para estabecer una forma única de clasificación, lo que es conocido es que cuanto más pequeña sea una partícula, mayor es su superficie en relación con su masa, con lo que con frecuencia, su reactividad y por tanto su toxicidad se ven tambien aumentadas; ya que la cantidad de átomos en contacto con la superficie es mayor. Si, además, la superficie de una partícula se ha modificado para lograr un determinado comportamiento, esto puede dar lugar a interacciones desconocidas con moléculas biológicas.

Debemos entender que la nanotecnología ofrece a la sociedad nuevas oportunidades de desarrollo y de mejora de la calidad de vida, pero esta convivencia con determinados 
nanomateriales, genera un marco nuevo de exposición doméstica y laboral poco explorado y de los que todavía se conoce poco en lo que respecta a los posibles efectos nocivos que puedan existir para la salud.

Se sabe muy poco de los efectos a corto y a largo plazo sobre la salud humana y el medio ambiente, aunque se ha observado que las nanopartículas artificiales pueden causar daños diferentes a los que producen las sustancias naturales. Esto es debido a que las propiedades de las sustancias en su estado natural (muchas ya conocidas), son diferentes cuando la materia se presenta a esta escala tan pequeña, comportándose de modo diferente en el cuerpo. Su diminuto tamaño, facilita su absorción respiratoria y a través de la piel, circulando por el flujo sanguíneo y permitiendo así su penetración en órganos y tejidos "cerrados" para esas mismas sustancias en otros tamaños.

Por otra parte, las pruebas de toxicidad tradicionales parecen no funcionar de modo adecuado respecto a los nanomateriales, como puede ser en el caso de la exposición a CNT. Además, este tipo de pruebas servirían sólo para toxicidades a corto plazo y no para anticipar posibles efectos a largo plazo como pueden ser los efectos cancerígenos o mutagénicos.

Los posibles efectos sobre la salud pueden estar relacionados con el número de partículas y la superficie de exposición, pero otras características como la forma, carga, componente químico, propiedades catalíticas, asociación a otros contaminates, grado de aglomeración y solubilidad en los fluídos biológicos, podrían influir de forma decisiva en la respuesta biológica. En este sentido, el grado de solubilidad en fluidos biológicos parece ser un factor importante, y dependiendo de su composición química, algunas NPs pueden disolverse más rápidamente que otras en los fluidos biológicos y adquirir propiedades tóxicas de carácter sistémico, además de sus posibles efectos locales.

Aunque se han realizado diferentes estudios "in vitro $e$ in vivo" en diferentes especies animales para determinar la toxicidad de las NPs, los conocimientos actuales se centran más en las reacciones biológicas frente a metales de transición como el silicio, el carbono, óxidos de metal y unos cuantos agentes, que han sido seleccionados como potenciales vectores de los agentes farmacéuticos. Sin embargo, a pesar del número creciente de industrias y poblaciones expuestas, todavía existe gran desconocimiento sobre aspectos toxicológicos relacionados con las NPs, así como los sistemas de vigilancia de la salud y los programas de higiene industrial más eficaces dentro del sector de la nanotecnología.

Nuestro objetivo se centra en revisar y evaluar la literatura científica para disponer del conocimiento más actual, en cuanto a evidencias, sobre relación que pueda existir entre la exposición a NPs y sus posibles efectos tóxicos y daños sobre la salud.

\section{METODOLOGÍA}

Se realizó una búsqueda en las principales bases de datos bibliográficas del ambito de la biomedicina, entre las que se seleccionaron MEDLINE (a través de Pubmed), OSH UPDATE, LILACS, SCIELO, CISDOC e Índice Bibliográfico Español de Ciencias de la Salud (IBECS).

Para establecer la estrategia de búsqueda se utilizaron los siguientes términos MeSH: Nanoparticles, Nanotechnology, Occupational Health, Occupational Disease, Riesgos Laborales, Nanocomposite, Nanoclay, Nanospheres, Fullerenes, Carbon Nanotube (Tabla III). 
Tabla III. Listado de los diferentes términos de búsqueda empleados en las diferentes bases de datos y el número de artículos obtenidos

\begin{tabular}{|c|c|c|c|}
\hline Base de Datos & Descriptores & Límites & N. ${ }^{\circ}$ Artículos \\
\hline \multirow{6}{*}{ MEDLINE } & Nanoparticles & Sin & 0 \\
\hline & Nanotechnology & Sin & 0 \\
\hline & Nanotechnology and Occupational Health & Sin & 34 \\
\hline & Nanotechnology and Occupational Disease & $\operatorname{Sin}$ & 10 \\
\hline & Nanoparticles and workplace & Sin & 18 \\
\hline & Nanocomposite and Occupational & Sin & 0 \\
\hline \multirow{6}{*}{ IBECS } & Nanotecnología y exposición & Sin & 1 \\
\hline & Enfermedades profesionales & Sin & 0 \\
\hline & Nanomateriales & Sin & 0 \\
\hline & Nanotecnología & Sin & 27 \\
\hline & Nanotecnología y ocupacional & $\operatorname{Sin}$ & 2 \\
\hline & Nanotechnology and occupational & Sin & 2 \\
\hline \multirow[t]{2}{*}{ LILACS } & Nanotecnología & Sin & 64 \\
\hline & Nanotecnología y salud ocupacional & Sin & 1 \\
\hline \multirow[t]{4}{*}{ SCIELO } & Nanotecnología y enfermedad profesional & Sin & 0 \\
\hline & Nanotecnología & Sin & 6 \\
\hline & Nanotechnology & Sin & 4 \\
\hline & Nanoparticles and occupational health & Sin & 0 \\
\hline \multirow[t]{2}{*}{ OSH UPDATE } & Nanotechnology and occupational health & Descriptores & 46 \\
\hline & Nanotechnolgy and occupational disease & Descriptores & 5 \\
\hline \multirow[t]{2}{*}{ CISDOC } & Nanotecnología & & 41 \\
\hline & Nanotecnología y enfermedades profesionales & & 15 \\
\hline TOTAL ARTÍCULC & & & 222 \\
\hline
\end{tabular}

Se completó esta búsqueda con documentos obtenidos de instituciones europeas y americanas dedicadas a la Salud y Seguridad en el Trabajo, entre las que cabe destacar el Instituto Nacional de Seguridad y Salud en el Trabajo (NIOSH), OSHA Europa y OSHA Estados Unidos en sus áreas de nanotecnología, el portal gubernamental de las nanotecnologías de USA ${ }^{3}$, portal francés específico de nanotecnología ${ }^{4}$,el Instituto Nacional de Seguridad e Higiene del Trabajo (INSHT) ${ }^{5}$ de España, el Instituto Riojano de Salud Laboral $^{6}{ }^{\mathrm{y}}$ agentes sociales como ISTAS-CCOO ${ }^{7}$.

La última fecha de búsqueda fue el 10/12/2012.

\section{Selección de artículos}

Se realizó una primera selección de los artículos obtenidos en base a la revisión de resúmenes de los mismos, atendiendo a criterios de pertinencia. A la colección resultante se le aplicaron los siguientes criteros de inclusión y exclusión:

Criterios de inclusión:

- Publicaciones dirigidas al estudio de riesgos de la nanotecnología y posibles enfermedades derivadas de la exposición a nanopartículas.

- Estudios publicados en inglés y español.

- Estudios nacionales e internacionales.

Criterios de exclusión:

- Artículos no relacionados con exposición laboral y/o toxicidad de nanopartículas.

- Se rechazaron aquellos artículos identificados como publicación duplicada, incluyéndose en el análisis el estudio más completo.

El nivel de evidencia se estableció en base a los crieterios del Scottish Intercollegiate Guidelines (SIGN) ${ }^{8}$. 


\section{RESULTADOS}

A partir de la estrategia de búsqueda establecida se obtubo una primera colección de 222 artículos, que fue depurada eliminando duplicados y aplicando criterios de pertinencia, así como los criterios de inclusión y exclusión establecidos previamente, quedando 39 artículos que cumplían los criterios requeridos para el estudio; la mayoría obtenidos a través de OSH UPDATE (45.7\%) y MEDLINE (40.2\%), seguidos de CISDOC (5.7\%), LILACS, IBECS y SciELO (2.8\% cada uno).

De estos 39 trabajos seleccionados, 28 se correspondían con artículos de revisión, por lo que no se incluyeron en el estudio de evaluación de resultados procedentes de trabajos originales, aunque si se tuvieron en cuenta a la hora de elaborar los apartados de discusión y conclusiones. Finalmente quedaron 11 artículos seleccionados para el estudio. De ellos, 9 tratan sobre posibles efectos sobre la salud de la exposición laboral a nanopartículas y partículas ultrafinas, 1 reproduce la exposición a partículas ultrafinas en ambiente laboral en laboratorio y 1 estudia la toxicidad cutánea por nanopartículas de Ag no laboral (Tablas IV y V).

\section{Principales hallazgos}

Song Y. \& col. (2009) ${ }^{9-11}$. Los autores consideran que se trata de la primera descripción de un caso laboral descrito en la literatura científica. Se trata de 8 trabajadores ( 7 mujeres y 1 hombre) de un departamento de pinturas decorativas y de impresión de una fábrica de China, confinados en un espacio de $70 \mathrm{~m}^{2}$, sin ventanas y con un único extractor de aire que no funcionaba desde 5 meses antes de que apareciesen los primeros síntomas. A diario se encargaban de manera rotatoria de la preparación, manipulación, calentamiento y aerosolización de una pasta que mezclaba polvo de marfil blanco con un éster poliacrílico ${ }^{9}$ (que contenía NPs de sílice y nanosilicatos) ${ }^{10,11}$. Su único equipamiento protector individual era una máscara de gasa de algodón que usaban ocasionalmente.

Tras un periodo que oscila entre 5 y 18 meses, todas las mujeres comenzaron a presentar la misma sintomatología respiratoria. El único varón expuesto era un trabajador temporal durante 3 meses que estaba asintomático. Las mujeres, de edades comprendidas entre 18-47 años, presentaban disnea respiratoria y derrame pleural recurrente de gran tamaño. Asimismo, todas ellas relatan que durante el proceso de aerosolización presentaban una erupción cutánea pruriginosa en su cara, manos y antebrazos. Además de dicha toxicidad por vía dérmica, es en ese momento cuando las NPs inhaladas pueden penetrar en los alvéolos pulmonares, donde los macrófagos, tras fagocitarlas las transportarían hasta el tejido pulmonar intersticial, en el cual pudo observarse, en todas las trabajadoras, una inflamación pulmonar inespecífica con material proteináceo que evolucionó a fibrosis pulmonar y granulomas pleurales de cuerpo extraño.

Desde el tejido pulmonar las NPs pueden llegar a la pleura y resto de órganos, vía sanguínea y linfática, produciendo la patología multiorgánica que presentaban las trabajadoras ( 5 de ellas padecieron derrame pericárdico y 3 adenopatías linfáticas). Se observaron alteraciones en los test funcionales de pulmón presentando un patrón restrictivo ventilatorio, con daño pulmonar severo en 3 de los casos e hipoxemia normocápnica en 4 casos. También se constató daños a nivel hepático (3 presentaron hipertransaminasemia), renal (6 hipoproteinemia) y sanguíneo (5 monocitosis, neutropenia y elevación del índice de sedimentación eritrocitaria y 1 trombocitopenia).

Entre los 18 y 21 meses del comienzo de la sintomatología murieron dos trabajadoras por fibrosis pulmonar rápidamente progresiva. Su tiempo de exposición al tóxico oscilo entre 10 y 13 meses. Mediante microscopía electrónica de transmisión se aislaron agregados de nanosílica y/o nanosilicatos de $30 \mathrm{~nm}$ de diámetro en el exudado pleural y citoplasma, tanto de las células mesoteliales del derrame pleural, como de las propias células epiteliales del tejido pulmonar y de las sanguíneas del intersticio pulmonar. Todas ellas se asemejaban a células apoptóticas con la cromatina condensada y marginalizada en forma de media luna ${ }^{9,11}$. 
Cheng TH \& col. $2012^{12}$. Los autores de este artículo describen un caso de exposición ocupacional a dióxido de titanio con resultado de muerte. Se trataba de un varón de 58 años, con patología de base (diabetes mellitus y cirrosis alcohólica de grado Child-Pugh A, estable en el momento en que se produjo la exposición). Después de desempeñar labores de aerosolización durante 3 meses en una fábrica de pintura que contenía polvos de poliéster de titanio, ingresaba en el hospital por fiebre, ictericia conjuntival y bronquiolitis obliterante precisando ventilación mecánica al sexto día del ingreso.

La radiografía de tórax y el TAC mostraron consolidación bilateral del espacio aéreo y patrón en "vidrio esmerilado". Según los resultados anatomopatológicos obtenidos en la biopsia pulmonar practicada en el octavo día post-ingreso, fue diagnosticado y tratado de Neumonía organizada. Su estado continuaba deteriorándose hasta producirse el fallecimiento por septicemia refractaria un mes posterior a su ingreso.

El la visualización de tejidos mediante microscopía electrónica de transmisión se identificaron partículas de dióxido de titanio y de sílice de 100-300 nm presentes en las muestras pulmonares. Las partículas de dióxido de sílice fueron descartadas como causa probable de esta neumonía organizada por falta de concordancia con los hallazgos anatomopatológicos mostrados en la biopsia de tejido pulmonar (inflamación pulmonar parenquimatosa con tejido de granulación en los conductos alveolares y alveolos).

Baxter CS \& col. 2010 ${ }^{13}$. Este estudio realiza, por primera vez, una detallada investigación del tamaño y concentración de las PUFs liberadas durante la ignición de 7 escenarios a los que habitualmente se enfrentan los bomberos en su trabajo (cocina, habitación, salón, cubiertas de la casa de madera y cemento, ático e interior de un coche), reproducidos en un laboratorio, puesto que la exposición a PUFs respiradas podría ser considerada un importante factor de riesgo de patología coronaria, pudiendo llegar a producir infarto agudo de miocardio. La medición se realizó aisladamente en cada uno de los escenarios cada 67 segundos, durante 30 minutos, con un espectrómetro de partículas de amplio rango, capaz de medir partículas de rangos comprendidos entre 0.01 hasta 10 micras $(10 \mathrm{~nm}$ hasta $10.000 \mathrm{~nm})$, situado a la misma altura y posición en todas la pruebas. Las PUFs liberadas en cualquiera de los escenarios fueron las más prevalentes, estando en más del 70\% comprendidas en el rango de tamaño 0,01-0.11 micras (10-110 $\mathrm{nm}$ ), alcanzándose altas concentraciones de las mismas en el salón y ático, llegando en el caso de la cocina y habitación a ser respectivamente 200 y 400 veces superior a las medidas en condiciones basales; mientras que en el coche las concentraciones detectadas fueron las más bajas (sólo 2 veces por encima de la situación basal). Entendemos por situación basal las concentraciones de PUFs obtenidas en condiciones de normalidad, sin estímulo externo alguno. Todas estas concentraciones fueron detectadas en el momento de revisión del área apagada, por colapso del espectrómetro durante la fase inicial de supresión del fuego con agua. No obstante, en el ático las PUFs liberadas son de 0.1 micras $(100 \mathrm{~nm})$ ó menos, detectándose tanto en la fase de apagado del fuego (después de 9,12 minutos tras la ignición), como de revisión (13,43 minutos después de la ignición) y continúan hasta el final de la medición. La exposición a PUFs que podría ocasionar patología cardiaca, debiera ser analizada como factor de riesgo ocupacional adicional en los bomberos, especialmente durante la fase de revisión de la zona quemada, momento en el cual se suelen desprender de las mascarillas respiratorias protectoras.

Maoxin Wu y Ronald E. Gordon \& col. $2009^{14}$. En este artículo los autores describen los hallazgos clínicos, anatomopatológicos y de minerales en siete trabajadores previamente sanos que se enrolaron en el programa de control y tratamiento médico del Hospital Monte Sinaí para los trabajadores del rescate de las Torres Gemelas tras los atentados ocurridos el 11 de Septiembre del 2001 en Nueva York, en el que una densa nube de humo con altos niveles de contaminantes transportados por el aire, cubrió Manhattan y partes de Brooklyn. Entre 60.000 y 70.000 profesionales de intervención inmediata resultaron expuestos. A partir de esta fecha se comprobó que muchos de los trabajadores expuestos desarrollaron algún tipo de patología, principalmente trastornos de vías respiratorias altas y bajas. Los signos y síntomas más frecuentes fueron tos 
persistente, disnea, sibilancias, asma, sinusitis, laringitis y asma inducida por irritantes. También se encontraron algunos casos de enfermedad intersticial pulmonar, incluyendo neumonía eosinofílica aguda, neumonitis granulomatosa, sarcoidosis y bronquiolitis obliterante. Los siete trabajadores del estudio, estuvieron expuestos al polvo del derrumbamiento de las torres los días 11 y/o 12 de septiembre de 2001 y sufrían una insuficiencia respiratoria severa o hallazgos radiológicos inexplicables. Se les realizó una biopsia pulmonar dirigida por videotoracoscopia. Se examinaron también muestras del polvo del lugar del derrumbamiento. En algunos de estos trabajadores con enfermedad pulmonar persistente se encontró nanotubos de carbono (NTC) en la biopsia pulmonar con confirmación en las muestras de polvo. Los NTC estaban presentes en 3 de 4 trabajadores que presentaban anormalidades parenquimatosas/intersticiales severas y en uno de dos trabajadores con enfermedad de las vías respiratorias pequeñas. Los autores señalan que el hallazgo de NTC en biopsias pulmonares y muestras de polvo del lugar, no era esperable y que requería de más estudios.

James I. Phillips \& col. $2010^{15}$. Los autores de este artículo reexaminan un caso previamente informado ${ }^{15}$ de exposición ocupacional a NPs de níquel con resultado de fallecimiento de un trabajador. La muerte por Síndrome de Distress Respiratorio del Adulto (SDRA) y los aspectos de higiene ocupacional del caso, fueron descritos tras la reconstrucción de las condiciones en las que se operaba en un proceso de niquelado por arco metálico en $1994^{16}$. Se realizó un examen minucioso de las muestras patológicas y el tejido pulmonar en búsqueda de níquel y se identificaron partículas de níquel de menos de $25 \mathrm{~nm}$ de diámetro en macrófagos pulmonares usando un microscopio electrónico de transmisión (TEM). El resto de las partículas podrían haber sido eliminadas y la presencia de níquel en orina podría apoyar la teoría de que las NPs inhaladas pueden viajar por el cuerpo y afectar a otros órganos. El nivel de níquel en orina en el trabajador fue de 780 $\mathrm{mg} / \mathrm{l}$, un nivel tan alto como el que se encuentra en los trabajadores de las refinerías de níquel y de 60 a 200 veces más alto que el encontrado en los soldadores de los astilleros. Además se encontraron hematuria, proteinuria y evidencia histológica de necrosis tubular en el riñón. Este estudio indica que las NPs de níquel pueden ser un peligro toxicológico y causar enfermedad aguda y fatal en humanos. Se midieron altos niveles de níquel en orina y sus riñones mostraban signos de Necrosis Tubular Aguda. Las condiciones en las cuales el hombre había llevado a cabo el proceso fueron reconstruidas en el ambiente de la planta por técnicos de higiene llevando cascos protectores y respiradores. No se encontraron daños para la salud cuando se recreó el pulverizado de níquel mediante arco metálico y se utilizaban equipos protectores adecuados. Las NPs deben ser usadas con precaución y especial atención debe ser prestada al uso de protección respiratoria, sobre todo cuando existe exposición en forma de aerosol (spray).

Elihn K. \& col, $2008{ }^{17}$. Este estudio en Suecia forma parte de una investigación epidemiológica que encontró que los trabajadores del asfalto muestran mayor pérdida de función pulmonar $\left(\mathrm{FEV}_{1}\right.$ y $\mathrm{FEF}_{50}$ disminuidos) que un grupo de referencia de trabajadores de la construcción ${ }^{18}$ y que durante la temporada de asfaltado muestran un aumento significativo de los marcadores de la inflamación (Interleukina-6) ${ }^{19}$. La finalidad del estudio fue investigar los posibles agentes causales de la reacción inflamatoria, con énfasis especial en las PUFs. La toma de muestras se realizó entre abril y octubre de 2005 y 2006 para medir la exposición a polvo total y gases de los asfaltadores y los productores de asfalto a través de muestreos personales en 51 trabajadores. Las mediciones de carbón particulado, tanto elemental como orgánico, se llevaron a cabo con muestreadores de aerosoles. La evaluación de la exposición a PUFs se realizo con contadores de partículas y un medidor de tamaño de partículas tipo nanoScan SMPS que iba dentro de una furgoneta. Los medidores de tamaño de NPs son pesados y necesitan electricidad por lo que no hubo posibilidad de llevar a cabo muestreos personales de PUFs. La mayoría de los niveles de exposición del equipo de asfaltado fueron de ligeras a moderadas con respecto a los niveles límites de exposición en Suecia para $\mathrm{NO}_{2}$, polvo total y carbón elemental. Los niveles de vapores de aceite sobrepasaron los límites de exposición laboral en algunas ocasiones. Los asfaltadores y los trabajadores de las plantas de asfalto estaban expuestos a PUFs en concentraciones medias de $3.4 \times 10^{4} / \mathrm{cm}^{3}$. En la 
zona de asfaltado el porcentaje de PUFs (por debajo de $100 \mathrm{~nm}$ ) fue de un $74 \%$. La concentración basal fue de $7.000 \mathrm{~cm}^{-3}$. Los niveles pico durante el pavimentado frecuentemente llegaron a $1 \times 10^{5}-2,2 \times 10^{5} \mathrm{~cm}^{-3}$. En la planta de producción de asfalto los niveles pico fueron similares a los del equipo de asfaltado por lo que se concluyó que el asfalto caliente por si mismo puede generar altas concentraciones de PUFs. Las partículas de la planta de producción de asfalto fueron más grandes que las de la zona de asfaltado, unos $180 \mathrm{~nm}$, posiblemente debido a que la temperatura del asfalto era más alta $\left(160^{\circ} \mathrm{C}\right)$. Los niveles basales fueron de $4500 \mathrm{~cm}^{-3}$ que se pueden comparar con la concentración de partículas encontradas en un paraje rural a las afueras de Estocolmo. Los equipos de medida actuales además de no resultar adecuados para el muestreo personal, como ya se ha señalado, tampoco permiten discriminar entre las PUFs de fondo y las generadas por el proceso estudiado. Los asfaltadores estaban expuestos a altas concentraciones de PUFs durante su jornada de trabajo lo que podría estar relacionado con los efectos adversos observados en la salud de estos trabajadores.

Frank E. Pfefferkorn \& col, $2010^{20}$. Estudiaron la posible emisión de partículas en aerosol desde la nanoescala (menor de $100 \mathrm{~nm}$ ) a ultrafinas $(<1 \mathrm{~mm})$ durante la soldadura de fricción de 2 aleaciones de Aluminio en un entorno de laboratorio y caracterización de su composición físico-química, ya que este tipo de soldadura se considera uno de los mayores progresos tecnológicos de los últimos 50 años, por lo que sus aplicaciones industriales están creciendo rápidamente y por lo tanto, también el número de trabajadores que utilizan esta tecnología. Hasta el momento, no había informes sobre exposiciones aéreas durante este proceso, pero los autores encuentran que la soldadura de fricción genera altas concentraciones de PUFs y submicrométricas. La distribución del tamaño fue bimodal con 2 máximos a 30 y $550 \mathrm{~nm}$. El promedio diario de concentraciones específicas de metales fue $2.0(\mathrm{Zn}), 1.4$ (Al), y $0.24(\mathrm{Fe}) \mu \mathrm{g} \mathrm{m} \mathrm{m}^{-3}$. La sección de soldadura de fricción está equipada con 2 tubos de aspiración de humo que son de uso común en las instalaciones de soldadura. La concentración total media de partículas medida con los tubos de aspiración en funcionamiento no se podía distinguir de la del valor basal. Por lo tanto el sistema de ventilación fue efectivo para reducir la exposición a NPs emitidas por este tipo de soldadura. Existe la posibilidad de que se produzcan exposiciones importantes a PUFs durante la soldadura de fricción por lo que proponen que se deben investigar en mayor profundidad las posibles exposiciones y sus efectos, especialmente en operaciones industriales a larga escala.

Trop \& col en $2006^{21}$.Describe el caso de un joven con quemaduras en el $30 \%$ del cuerpo que tras un semana de tratamiento local con vendajes con plata, presenta síntomas de hepatotoxicidad y coloración grisácea en la cara, encontrándose niveles de plata en orina y plasma elevados. Estos niveles se normalizaron tras cese de la exposición.

Toyama T \& col, $2008^{22}$. Describe sobre un caso de eritema multiforme, descrito como respuesta alérgica tipo dermatitis de contacto en una persona con exposición laboral a dendrímeros en un laboratorio de investigación, cuya sintomatología reaparece tras la reincorporación al puesto de trabajo. 


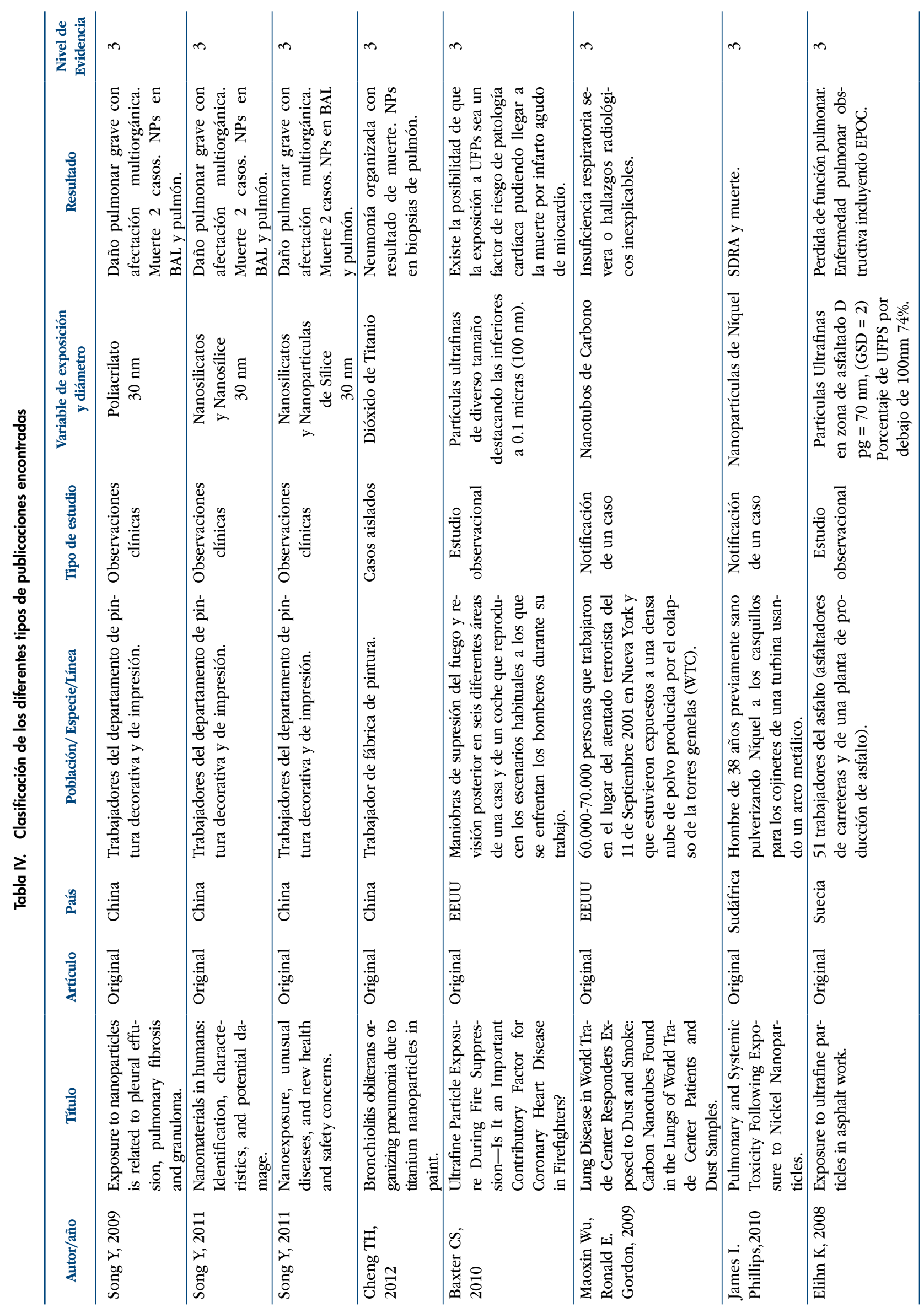




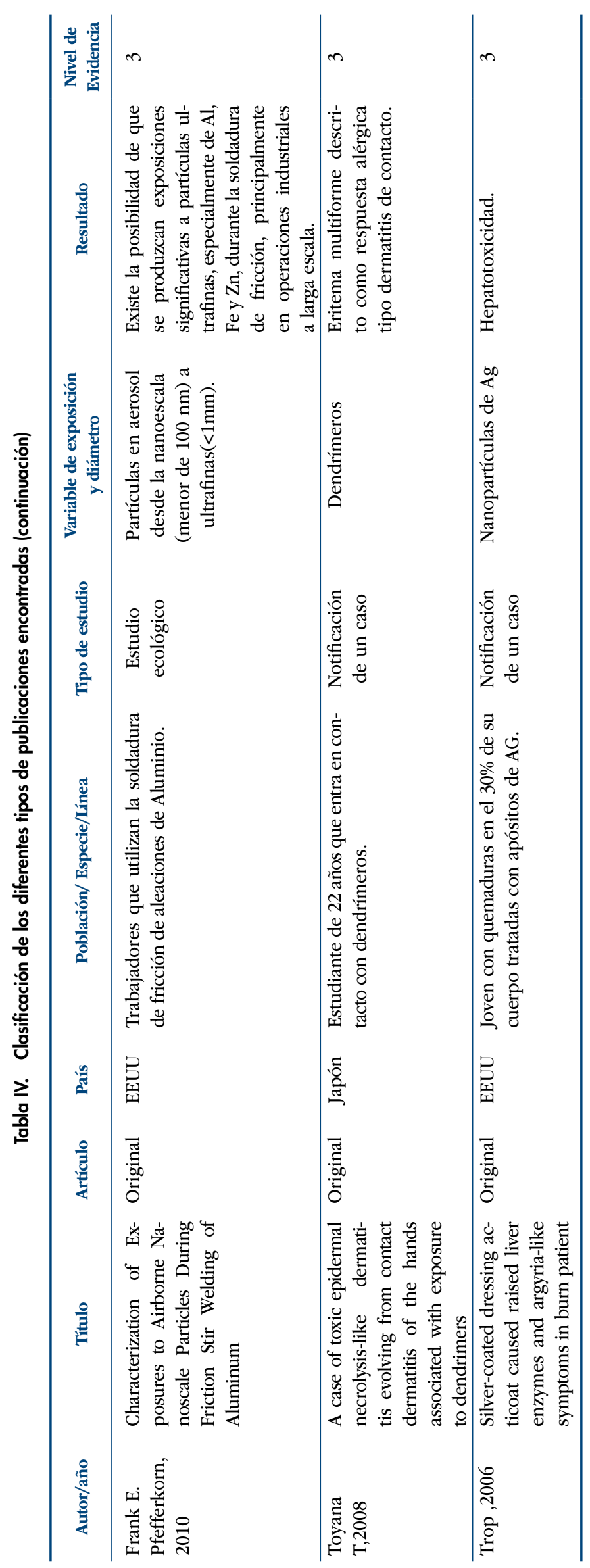









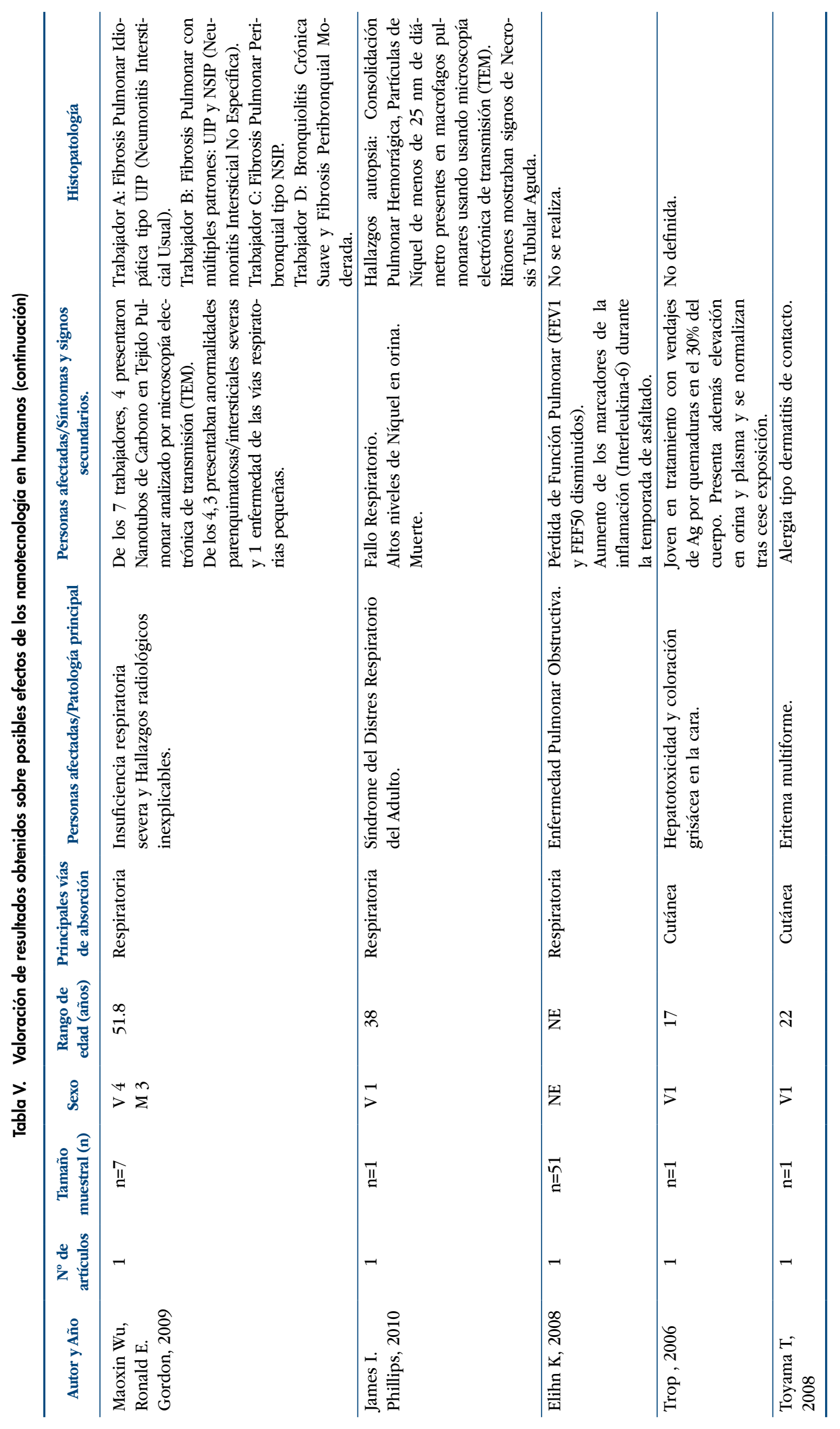




\section{DISCUSIÓN}

Existen muy pocas publicaciones que estudien la posible relación entre la exposición laboral a nanopartículas y la aparición de daño para la salud. Solamente encontramos 11 artículos publicados que componen nuestro estudio, por lo que es difícil establecer causalidad entre la exposición a nanopartículas y un daño sobre la salud; especialmente, porque en algunos artículos, los trabajadores habían estado expuestos también a otras sustancias y/o agentes externos, y ninguno de estos trabajos contaba con un diseño como estudio analítico.

Los estudios revisados son de muestras pequeñas, cuatro de los 10 artículos son casos informados $(n=1)^{12,15,21,22}$, y el resto son series de casos (de $7^{14} 8^{9,11}$ y $51^{17}$ casos) o estudios de exposición ${ }^{13,20}$. El nivel de evidencia de todos es tipo 3, que es el más bajo después de la opinión de expertos.

Aunque los mecanismos de toxicidad no están todavía elucidados para la mayoría de las nanopartículas, se consideran entre otros, la formación de especies reactivas de oxígeno, que liberarían radicales libres tóxicos y el desarrollo de estrés oxidativo. Ambos desencadenarían una respuesta inflamatoria local, que vía de activación de la cascada de señales celulares, puede promover el daño celular y tisular con respuestas fibróticas en los órganos afectados, cambios histologicos que son asociados con enfermedad en el órgano diana ${ }^{23}$.

A pesar de estas limitaciones en los datos disponibles en humanos, algunos estudios en animales han encontrado que los NTC pueden producir respuestas inflamatorias, inmunes, fibrogénicas y granulomatosas en ratas y ratones en función de la cantidad de nanofibras y la vía de administración ${ }^{24}$.

Agentes de exposición, la evidencia disponible sobre el comportamiento de las NPs en los sistemas biológicos se limita principalmente a los metales de transición como silicio y carbono (nanotubos, fullerenos), a óxidos de metales y unos pocos agentes, que han sido seleccionados como vectores potenciales de agentes farmacológicos.

Vías de exposición y posible etiopatogenia del daño tisular, la exposición humana a nanopartículas ocurre principalmente por vía inhalatoria, digestiva, cutánea y parenteral $^{25}$.

Vía inhalatoria, los efectos tóxicos de las nanoparticulas a nivel respiratorio han sido los más estudiados hasta el momento. Una vez que las nanopartículas son inhaladas, el 50\% de las nanopartículas menores de 15-20 nm se depositan a nivel alveolar donde son fagocitados por los macrófagos; mientras que las menores de $10 \mathrm{~nm}$, se depositan principalmente en la región extratorácica ${ }^{25}$.

Debido a que el sistema respiratorio es un blanco único para la toxicidad de las nanopartículas y que además de ser la puerta de entrada para las partículas inhaladas, recibe el gasto cardíaco completo, existe la posibilidad de que la exposición del pulmón a las nanopartículas que entran en el cuerpo a través de la respiración o cualquier otra vía, pueda acabar en una distribución sistémica de las mismas ${ }^{26,27}$. Por esto, debe dedicarse especial atención a la utilización de protección respiratoria, sobre todo cuando las partículas son aplicadas en forma de aerosol (spray), lo que de alguna forma queda confirmado por los resultados de Phillips et al., al reproducir pulverizado de níquel mediante arco metálico y comprobando que no se encontraban daños para la salud al utilizar equipos protectores adecuados ${ }^{15}$.

Los primeros estudios que investigaron la toxicidad de las NPs se enfocaron en la exposición atmosférica de los humanos a mezclas heterogeneas medioambientales de PUFs cuyos diámetros alcanzaran rangos nanométricos $(<100 \mathrm{~nm})$. Estos estudios examinaron la toxicidad pulmonar asociada al depósito de estas partículas en el tracto respiratorio de los organismos objeto del estudio. La densidad de PUFs en aire urbano alcanza rangos que oscilan de 5.000-30.000 partículas por centímetro cúbico. Estudios 
clínicos recientes, han puesto de relieve que niveles medios de 1,2 a $1,5 \times 10^{5} / \mathrm{cm}^{3}$ inducen en voluntarios sanos una gran variedad de cambios en los parámetros cardiovasculares ${ }^{13}$.

Algunos investigadores han encontrado un aumento del riesgo de asma infantil y del adulto en relación con la exposición a PUFs del aire de las ciudades. Sin embargo otros estudios no han encontrado dicha relación. Estudios de laboratorio con animales han mostrado rutinariamente un aumento en la inflamación pulmonar, estrés oxidativo y la afectación de órganos distantes después de la exposición respiratoria a PUFs inhaladas. Análisis de tejidos y cultivos celulares, también han apoyado la respuesta fisiológica vista en modelos animales y los datos obtenidos apuntan a un aumento de la incidencia de estrés oxidativo, la producción de citoquina inflamatoria y apoptosis en respuesta a la exposición a PUFs ${ }^{27}$.

Estudios epidemiológicos realizados en trabajadores expuestos a aerosoles, incluyendo PUFs y nanopartículas muestran disminución de la función pulmonar, síntomas respiratorios adversos, enfermedad pulmonar obstructiva crónica y fibrosis. Asimismo, el aumento de dichas partículas en la atmósfera se ha asociado a efectos cardiovasculares adversos en individuos susceptibles, como ancianos y pacientes con patología previa ${ }^{13}$.

Existe un interés creciente en las concentraciones de PUFs en los lugares de trabajo ${ }^{13,17,18,19,20}$, donde los trabajadores se ven expuestos a compuestos habitualmente mixtos, que al agregarse modifican sus características físico-químicas pudiendo ocasionar mayor severidad y precocidad en la aparición de patología asociada ${ }^{28}$.

A la luz de los estudios analizados en esta revisión parece encontrarse una posible relación entre la exposición a PUFs en determinados ambientes laborales y algunas patologías,especialmente, en trabajadores de la construcción durante la pavimentación de las carreteras $\left(2,2 \times 10^{5} / \mathrm{cm}^{3}\right)^{29}$, de plantas de producción de asfalto $\left(1,5 \times 10^{5} \text { a } 2,5 \times 10^{5} / \mathrm{cm}^{3}\right)^{17}$, extinción de incendios de estructuras domésticas (mayores de 4,95 x $10^{4}$ a 2,11 x $10^{6} / \mathrm{cm}^{3}$ ) ${ }^{13}$ y de automoción $\left(1,96 \times 10^{4} / \mathrm{cm}^{3}\right)$, además de entornos industriales relacionados con fundiciones, estrusionado, corte y laminado del aluminio ${ }^{13}$, ambientes de trabajo donde se llega a alcanzar en la sección de soldadura y de fricción niveles de $\left(4 \times 10^{5} / \mathrm{cm}^{3}\right)^{20}$. En todos ellos, los resultados medidos se encuentran en el rango superior de las concentraciones ambientales basales.

La pequeña variabilidad entre los estudios, puede ser explicada porque comparamos las PUFs liberadas al apagar el incendio de estructuras en su mayoría cerradas (habitaciones, ático, cocina, etc), que favorece la aparición de las concentraciones más altas descritas, frente a las determinadas durante el asfaltado de carreteras en un área rural de Suecia, en las que dependiendo de las condiciones climáticas (viento, calor, etc) y de la densidad del tráfico rodado en dicha carretera, se detectan concentraciones ligeramente inferiores.

Por todo ello, parece plausible considerar a las PUFs como un factor de riesgo añadido en determinados lugares de trabajo.

\section{Vía Dermatológica}

A parte de la vía inhalatoria, otra exposición muy frecuente en los trabajadores, es el contacto a través de la piel; por lo que la segunda vía más importante de entrada de NPs en el organismo es la vía cutánea ${ }^{29,30}$, bien por la presencia de NPs en el ambiente laboral que pueden llegar a depositarse en la piel, o bien, por el uso de cosméticos y cremas solares.

Se han realizado varios estudios tanto "in vivo e in vitro" para determinar si las nanopartículas se pueden absorber a través de la piel, produciendo efectos locales, o bien, daños sistémicos por su difusión.

Según M. Crosera ${ }^{29}$ hay pocos estudios sobre la penetración en la piel de las NPs y existen discrepancias en sus resultados, probablemente por diferentes técnicas y métodos 
empleados, condiciones de laboratorio y ausencia de protocolos de evaluación estandarizados. Son fundamentalmente estudios "in vivo e in vitro" sobre piel porcina y humana.

\section{Vía Digestiva}

Aunque la exposición oral a NPs, bien podría ser por ingestión de comida y agua contaminada, deglución de partículas inhaladas o por malas práctica higiénico-dietéticas durante el manejo de nanomateriales ${ }^{30}$, en nuestro estudio no se ha encontrado ningún artículo que refiera posibles efectos sobre la salud ante una posible exposición oral a NPs.

\section{Otras Vías}

Otras vías incluyen la exposición humana por efecto de la biomedicina, fundamentalmente por utilización de fármacos por vía parenteral ${ }^{30}$. Aunque sería muy arriesgado establecer algún tipo de relación, algunos trabajos en marcha, aún en fase muy preliminar, intentan estudiar posibles relaciones entre la presencia de NPs en algunos procedimientos diagnósticos o de tratamiento y la aparición de determinados tipos de cáncer.

En conclusión, la evidencia sobre exposición laboral a nanopartículas como factor de riesgo en el desarrollo de determinadas patologias es muy limitada. Sin embargo, estudios en animales de laboratorios, análisis de niveles de exposición y hallazgos anatomopatológicos en poblaciones expuestas, pueden sugerir una posible respuesta a nivel pulmonar y una toxicidad dermatológica ante la exposición a determinados tipos de NPs, teniendo en cuenta que aún son necesarios más estudios epidemiológicos para valorar el riesgo asociado a la exposición por inhalación o a través de la piel en trabajadores expuestos a estos materiales.

Entre otros grandes retos, parte de la dificultad para su realización se centra en la falta de metodos de screening validados para la toxicidad de los nanomateriales, de equipos para medir las exposiciones y de nanomateriales de referencia. Estos estudios deberian orientarse a determinar tanto si pudiera existir un efecto negativo de la exposición a NPs sobre la salud, como a identificar el tipo de sustancia o agente, la vía de exposición asociada al riesgo para la salud, y la dosis tóxica. Además, debería caracterizarse la respuesta del organismo ante estas partículas cuando son inhaladas, absorbidas a través de la piel, o ingeridas.

Con la escasa información disponible, mientras se desarrollan más trabajos orientados a estudiar en profundidad posibles relaciones causa-efecto entre patologías y exposiciones a diferentes NPs y se pueda determinar con mayor precisión si existe o no algún riesgo para la salud asociado con la exposición a estos agentes se debe: 1) mantener la precaución cuando se sospeche la posibilidad de exposición a NPs y 2) recomendar el establecimiento de medidas de prevención como una de las formas más eficaces con las que actualmente podemos contar para poder evitar el riesgo de desarrollar enfermedades profesionales posiblemente relacionadas con la exposición a NPs.

\section{REFERENCIAS BIBLIOGRÁFICAS}

1. Gutierrez-Praena et al. Nuevos riesgos tóxicos por exposición a nanopartículas. Revista de Toxicología, 2009; 26 (2-3): 87-92.

2. Vladimir Murashov, Paul Schulte \& John Howard (2012): Progression of Occupational Risk Management with Advances in Nanomaterials, Journal of Occupational and Environmental Hygiene, 9:1, D12-D22

3. National Nanotechnology Initiative. Official website of the United States National Nanotechnology Initiative. http://www.nano.gov/ 2012. 21-12-2012

4. INRS Occupational Health Research Conference 2011: Risks associated to Nanoparticles and Nanomaterials. http://www.inrs-nano2011.fr/ 2011. 17-12-2012

5. INSHT. Instituto Nacional de Seguridad e Higiene en el Trabajo. http://www.insht.es/InshtWeb/ Contenidos/Documentacion/FichasTecnicas/NTP/Ficheros/786a820/797\%20web.pdf. 2008. 17-12-2012

6. Instituto Riojano de Salud Laboral. Logroño 2011. La seguridad y salud en la exposición a nanopartículas. http://www.uv.es/cadep/documents/prevencion/Seguridad $\% 20 \% 20 y \% 20$ salud\%20nanoparticulas.pdf 2011. 16-12-2012 
7. ISTAS. Instituto Sindical de Trabajo, Ambiente y Salud. Peligros y riesgos laborales. Nanotecnología. http://www.istas.net/web/index.asp?idpagina=3332 2008. 17-12-2012.

8. Harbour R, Miller J, for the Scottish Intercollegiate Guidelines Network Grading Review Group. A new system for grading recommendations in evidence based guidelines. BMJ 2001; 323: 334-6.

9. Song Y. Exposure to nanoparticles is related to pleural effusion, pulmonary fibrosis and granuloma. European Respiratory Journal 2009; 34: 559-569.

10. Song Y. Nanomaterials in human: Identificacion, Characteristics and potencial damage. Toxicol Pathol 2011; 39 (5) 841-9.

11. Song Y, Tang S. Nanoexposure, Unusual Diseases, and New Health and Safety Concerns. The Scientific World Journal 2011; 11: 1821-1826.

12. Cheng TH et al. Bronchiolitis Obliterans Organizing Pneumonia Due in Titanium Nanoparticles in Paint. Ann Thoracic Surgery 2012; 93(2): 666-9.

13. Baxter CS et al. Ultrafine Particle Exposure During Fire Supression- Is it an important Contributory Factor for coronary Heart Disease in Firefignters. Journal of Occupational and Environmental Medicine 2010; 52(8): 791-796.

14. Wu M, Gordon RE, Herbert R, Padilla M, Moline J, Mendelson et al.Case report: Lung disease in World Trade Center responders exposed to dust and smoke: carbon nanotubes found in the lungs of World Trade Center patients and dust samples. Environ Health Perspect 2010;118(4):499-504.

15. Phillips JI, Green FY, Davies JC, Murray J. Pulmonary and systemic toxicity following exposure to nickel nanoparticles. Am J Ind Med. 2010;53(8):763-7.

16. Rendall RE, Phillips JI, Renton KA. 1994. Death following exposure to fine particulate nickel from a metal arc process. Ann Occup Hyg.1994;38(6):921-930

17. Elihn K, Ulvestad B, Hetland S, Wallen A, Randem BG. Exposure to ultrafine particles in asphalt work.J Occup Environ Hyg. 2008;5(12):771-9.

18. Ulvestad, B., Randem, B.G., Hetland, S., Sigurdardottir, G., Johannessen, E. and Lyberg, T. Exposure, lung function decline and systemic inflammatory response in asphalt workers. Scand. J. Work Environ. Health 2007;33(2):114-121.

19. Ulvestad, B., Randem, B.G., Barregard, L., Andersson, L. and Ellingsen, D. Clara cell protein as a biomarker for lung injury in asphalt workers. J. Occup. Environ. Med. 2007;49(10):1073-1078.

20. Pfefferkorn FE, Bello D, Haddad G, Park JY, Powell M, McCarthy J et al.Characterization of exposures to airborne nanoscale particles during friction stir welding of aluminum. Ann Occup Hyg. 2010;54(5):486-503.

21. Trop M, Novak M, Rodl S, Hellbom B, Kroell W, Goessler W. Silver-coated dressing acticoat caused raised liver enzymes and argyria-like symptoms in burn patient. J Trauma. 2006;60(3):648-652.

22. Toyama T, Matsuda H, Ishida I, Tani M, Kitaba S, Sano S et al. A case of toxic epidermal necrolysis-like dermatitis evolving from contact dermatitis of the hands associated with exposure to dendrimers. Contact Dermatitis 2008; 59(2): 122-123.

23. Johnston H, Pojana G, Zuin S, Jacobson NR, Meller P, Loft s, et al. Engineeered nanomaterial risk. Lessons learnt from completed nanotoxicology studies: potential solutions to current and future challenges. Crit Rev Toxicol 2013; 43(1): 1-20.

24. Bonner JC. Nanoparticles as a Potencial Cause of Pleural and Intersticial Lung Disease. Proceedings of the American Thoracic Society 2010; 7(2):138-41.

25. The Institut de recherche RobertSauvé en santé et en sécurité du travail (IRSST). Health Effects of Nanoparticles. http://www.irsst.qc.ca/media/documents/pubirsst/r-469.pdf 2006. 17-12-2012

26. Oberdörster G, Sharp Z, Atudorei V, Elder A, Gelein R, Lunts A, et al. Extrapulmonary translocation of ultrafine carbon particles following whole-body inhalation exposure of rats. J. Toxicol. Environ. Health Part A 2002; 65(20):1531-1543.

27. Costa R, Orriols R. Man-Made Mineral Fibers and the Respiratory Tract. Archivos de Bronconeumología (English Edition) 2012;48(12): 460-468.

28. Oberdörster G, Maynard A, Donaldson K, Castranova V, Fitzpatrick J, Ausman K et al. Principles for characterizing the potential human health effects from exposure to nanomaterials: elements of a screening strategy. Part Fibre Toxicol. 2005; 6(2): 1743-8977.

29. Crosera M, Bovenzi M, Maina G, Adami G, Zanette C, Florio C et al. Nanoparticle dermal absorption and toxicity: a review of the literature. Occupational Environ Health 2009;82(9); 1043-1055.

30. Forbe T, Garcia M, Gonzalez E. Potential risk of nanoparticles.Cienc.Tecnol. Aliment 2011;31 (4).

31. NIOSH. Current intellegence Bulletin 60: Interin guidance for medical screening and hazard surveillance for workers potentially exposed to engineered nanoparticles. http://www.cdc.gov/niosh/docs/2009-116/ pdfs/2009-116.pdf 2009. 20-01-13. 


\section{ANEXO A}

\section{Diferentes formas de nanopartículas}

\section{Fullerenos}
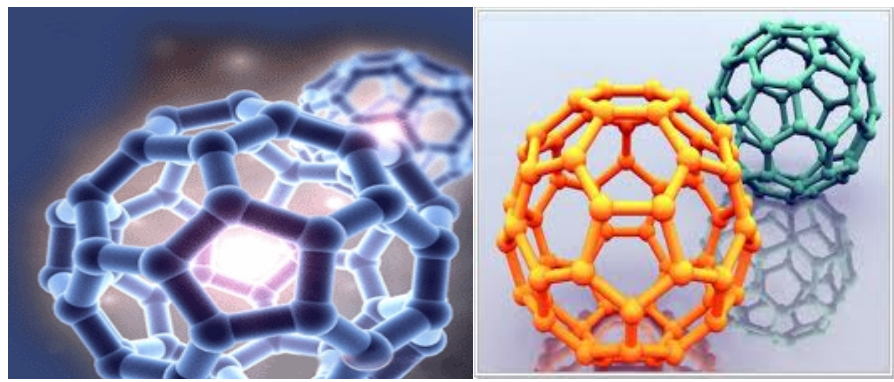

\section{Puntos cuánticos}

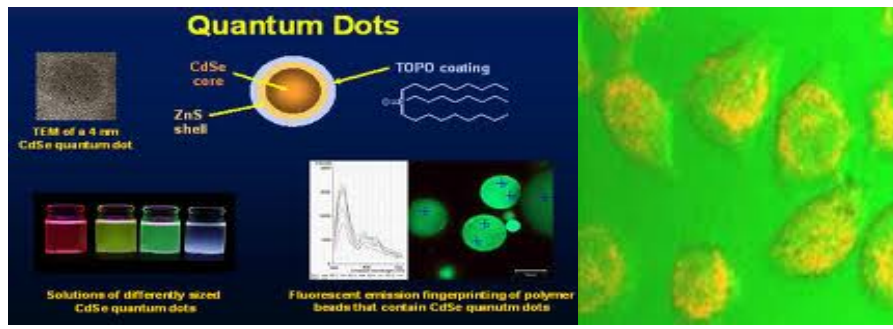

3. Nanotubos de carbono

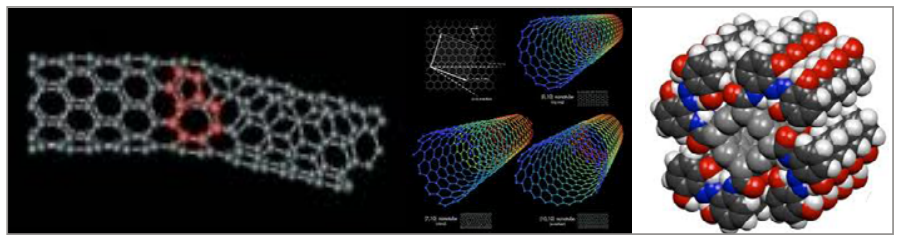

\section{Grafenos}

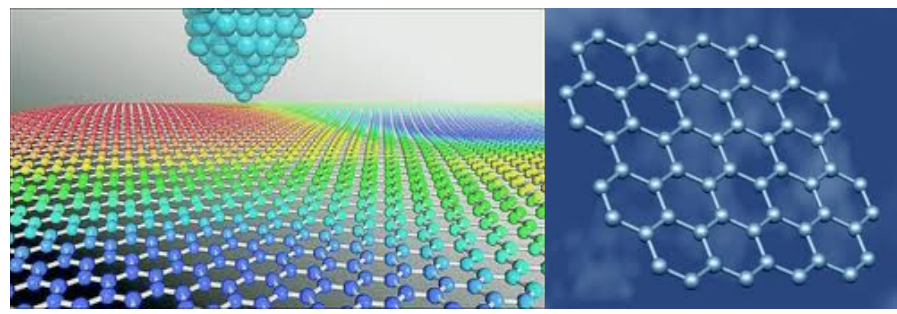

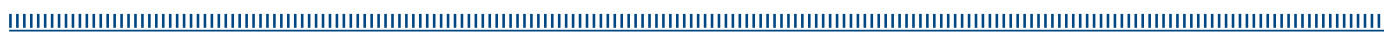

